Revta brasil. Bot., São Paulo, V.23, n.3, p.341-347, set. 2000

\title{
Comparação do padrão da distribuição espacial em escalas diferentes de espécies nativas do cerrado, em Brasília, DF
}

\author{
JOHN D. HAY ${ }^{1,2}$, MARCELO X. BIZERRIL ${ }^{1}$, ARMANDO M. CALOURO ${ }^{1}$, ELIZABETH \\ M.N. COSTA ${ }^{1}$, ANAMARIA A. FERREIRA ${ }^{1}$, MARIA L.A. GASTAL ${ }^{1}$, CÍCERO D. GOES \\ JUNIOR $^{1}$, DAGOBERTO J. MANZAN ${ }^{1}$, CLÁUDIO R. MARTINS ${ }^{1}$, JOICE M.G. MON- \\ TEIRO $^{1}$, SÉRGIO A. OLIVEIRA ${ }^{1}$, MARCELO C.M. RODRIGUES ${ }^{1}$, JOÃO A.S. \\ SEYFFARTH $^{1}$ e BRUNO M.T. WALTER ${ }^{1}$
}

(recebido em 1 de dezembro de 1998; aceito em 26 de abril de 2000)

\begin{abstract}
Comparison of scale in the spatial distribution of native species of the cerrado, em Brasília, DF). The scale of spatial distribution of three native tree species, Caryocar brasiliense, Pterodon pubescens and Sclerolobium paniculatum, and two native palm species, Syagrus comosa and $S$. flexuosa, in the cerrado was determined using three quadrat variance methods. The data were collected in an area of cerrado, sensu stricto, on the Fazenda Água Limpa of the University of Brasília in 128 contiguous quadrats of $5 \times 8 \mathrm{~m}$ each. In each quadrat, all individuals of each species were located and their height measured. The scale of spatial distribution of each species was evaluated with the following methods: blocked quadrat variance (BQV); two-term local quadrat variance (TTLQV) and paired quadrat variance $(\mathrm{PQV})$, for both all individuals and all woody individuals over $130 \mathrm{~cm}$ in height. The total number of individuals was 138 for $C$. brasiliense, 34 for $P$. pubescens, 82 for $S$. paniculatum, eight for $S$. comosa and 36 for $S$. flexuosa. All species showed a group in at least one of the methods. The time spent in collection of data was comparable to that for other studies of spatial distribuition and three of the five species had similar results in all methods. The pattern for $C$. brasiliense was similar to that found in another study using the same methodology, with a distance of approximately $350 \mathrm{~m}$ between groups.
\end{abstract}

RESUMO - (Comparação do padrão da distribuição espacial em escalas diferentes de espécies nativas do cerrado, em Brasília, DF). O padrão de agrupamento espacial de três espécies arbóreas, Caryocar brasiliense, Pterodon pubescens e Sclerolobium paniculatum, e duas espécies de palmeiras, Syagrus comosa e $S$. flexuosa, nativas do cerrado, foi investigado usando três métodos de variância entre parcelas. Os dados foram coletados em uma área de cerrado sensu strictu da Fazenda Água Limpa da Universidade de Brasília, em Brasília, DF, em 128 parcelas contíguas de 5 × $8 \mathrm{~m}$ cada. Em cada parcela, foram procurados todos os indivíduos das espécies, anotando o número encontrado e suas alturas. Os dados foram tabelados e analisados usando as técnicas de variância entre blocos de parcelas $(\mathrm{BQV})$, variância móvel entre blocos de parcelas (TTLQV) e variância entre parcelas pareadas (PQV), tanto para todos os indivíduos encontrados quanto para somente os indivíduos lenhosos maiores do que $130 \mathrm{~cm}$ em altura. O número total de indivíduos encontrado foi 138 para $C$. brasiliense, 34 para $P$. pubescens, 82 para S. paniculatum, oito para S. comosa e 36 para S. flexuosa. Todas as espécies apresentaram um padrão agrupado de distribuição espacial em pelo menos uma das metodologias e três das cinco espécies tiveram resultados semelhantes em todos os métodos. O padrão para $C$. brasiliense foi semelhante ao encontrado para esta mesma espécie em outra região do cerrado, utilizando a mesma metodologia, com uma distância de aproximadamente $350 \mathrm{~m}$ entre grupos.

Key words - Cerrado, spatial pattern, quadrat-variance methods

\section{Introdução}

A questão de escala em ecologia é muito importante (Levin 1992). Os padrões de distribuição espacial de uma espécie podem ser estudados em escala macro (biogeográfico), meso (comunidades) ou micro (distribuição espacial dos indivíduos dentro da comunidade). Além disto, é necessário distinguir entre a intensidade do padrão, relacionado com a variação na densidade dos indivíduos, e o grão, ou

1. Departamento de Ecologia, Universidade de Brasilia, Caixa Postal 04457, 70919-970 Brasília, DF.

2. Autor para correspondência: jhay@unb.br seja, a escala do padrão espacial destas diferenças em intensidade (Pielou 1969). Outrossim, a identificação de padrões espaciais em escala maior é importante para o planejamento e desenho de áreas de conservação (Hubbell \& Foster 1986). Para GriegSmith (1991), padrões em uma ou mais escalas são o resultado da heterogeneidade espacial de muitos fatores ambientais.

Em escala micro, uma espécie pode se distribuir no espaço de três formas básicas: aleatória, agrupada ou uniforme. Na distribuição aleatória, supõe-se uma homogeneidade ambiental levando a um comportamento não seletivo. Na distribuição agrupada, os indivíduos estão agregados nas partes mais favoráveis do habitat. Na distribuição uniforme, intera- 
ções negativas entre indivíduos (como, por exemplo, a competição por alimento ou espaço) resultam em espaçamento uniforme entre os indivíduos (Ludwig \& Reynolds 1988). Os principais fatores causais que podem levar às várias formas de distribuição não aleatória são: fatores ambientais (intensidade de luz, $\mathrm{pH}$, disponibilidade de água); reprodutivos (em decorrência da forma de reprodução ou dispersão de diásporos); sociais, devido ao comportamento inato (territorialismo); interações intraespecíficas (competição) e estocásticos, devido às variações aleatórias nos fatores citados anteriormente (Hutchinson 1953 apud Ludwig \& Reynolds 1988). Então, conhecer a maneira como os indivíduos de uma espécie se distribuem em uma comunidade é um dos primeiros passos para se entender a dinâmica de populações vegetais, sendo que na região dos cerrados faltam informações para a maioria das espécies.

O padrão dominante de espécies arbóreas nas savanas é a distribuição agrupada (San Jose et al. 1991). O mesmo parece ser verdadeiro para os cerrados, pois reunindo os dados existentes para 45 espécies do cerrado, 64\% tem distribuição espacial agrupada (J.D. Hay, dados não publicados).

Existem várias metodologias para determinar o padrão de distribuição espacial dos indivíduos em uma comunidade. Os métodos clássicos de análise de distribuição espacial baseiam-se em análise do número de indivíduos presentes em parcelas de tamanho fixo ou em distâncias entre indivíduos em uma comunidade (ver, por exemplo, Ludwig \& Reynolds 1988 e Brower et al. 1998). Todavia, nas metodologias em que se utilizam parcelas, o seu tamanho pode influenciar a conclusão, devido à relação entre o tamanho do indivíduo e o tamanho da parcela e a escala espacial da distribuição.

Um outro tipo de metodologia tenta superar o problema da influência do tamanho da parcela no resultado, através do uso de combinações de parcelas para acompanhar mudanças na variância em torno do número médio de indivíduos por parcela (Ludwig \& Reynolds 1988). Esta metodologia pode ser útil para detectar padrões na distribuição de indivíduos em escalas diferentes. Existem duas abordagens deste tipo de metodologia. Na primeira, denominada método de variância entre blocos de parcelas e aplicada pela primeira vez por Grieg-Smith (1952), o tamanho da parcela é aumentado através do agru- pamento de dados de parcelas contíguas. O segundo método, o de variância entre parcelas pareadas, foi desenvolvido por Goodall (1974) e Ludwig \& Goodall (1978) e baseia-se em comparações utilizando dados de distâncias diferentes entre parcelas. Estes métodos, embora não tão utilizados quanto os métodos clássicos, têm sido aplicados em estudos tanto do estrato rasteiro (Carter \& O'Connor 1991) quanto no arbóreo (Grieg-Smith 1991, Schaefer 1993, Stohlgren 1993).

O presente trabalho foi realizado com os objetivos de determinar o padrão de distribuição espacial de três espécies arbóreas e duas espécies de palmeiras, todas nativas do cerrado (sensu stricto), utilizando métodos de variância e verificando se há semelhança entre o padrão de distribuição para o conjunto total de indivíduos encontrados e o padrão do grupo de indivíduos maiores do que $130 \mathrm{~cm} \mathrm{de}$ altura.

\section{Material e métodos}

O trabalho de coleta de dados foi realizado no período de março a maio de 1993, em uma área de cerrado (sensu stricto) da Fazenda Água Limpa da Universidade Brasilia, Brasília, DF (1557'S e $\left.47^{\circ} 56^{\prime} \mathrm{W}\right)$. Foi estabelecida uma transeção de $640 \mathrm{~m}$ de comprimento por $8 \mathrm{~m}$ de largura, subdivida em parcelas menores de $5 \mathrm{~m}$ cada, num total de 128 parcelas contíguas de $8 \mathrm{x}$ $5 \mathrm{~m}$. Este tamanho de parcela foi adotado para facilitar a comparação dos dados obtidos neste estudo com os coletados por Dantas (1994) para Caryocar brasiliense. Em cada parcela, todos os indivíduos das espécies Caryocar brasiliense Camb. (pequí), Pterodon pubescens Vog. (sucupira branca) e Sclerolobium paniculatum Vog. var. subvelutinum Benth. (carvoeiro) foram contados e medidos quanto à sua altura, circunferência a $30 \mathrm{~cm}$ do solo, circunferência a $130 \mathrm{~cm}$ do solo e diâmetro da copa (eixo maior e menor). Caules separados espacialmente a uma distância inferior a $30 \mathrm{~cm}$ entre um e outro foram considerados como sendo o mesmo indivíduo. Além destas espécies lenhosas, todos os indivíduos das palmeiras Syagrus comosa Mart. e S. flexuosa Mart. encontrados nas parcelas também foram contados. Todas estas espécies são nativas e comuns ou abundantes no ecossistema do cerrado (Ratter et al. 1996).

O padrão da escala de distribuição espacial foi estabelecido usando métodos de variância, através da construção de gráficos nos quais se plota o valor da variância contra o tamanho do bloco ou intervalo entre parcelas (Ludwig \& Reynolds 1988). Os tipos de distribuição possíveis são aleatória, agrupada e uniforme. Determina-se uma distribuição aleatória, quando os valores de variância oscilam em torno da média do número de indivíduos por parcela. A distribuição agrupada ocorre quando há observação de um ou mais picos. A altura do pico, comparada com os outros valores, indica a intensidade do agrupamento. A presença de mais de um pico indica várias escalas de agrupamento da espécie. A distância entre os grupos é dada através da multiplicação do valor 
do número do bloco onde foi observado o pico por dois e, também, pelo comprimento da parcela, o qual neste estudo é cinco. A distribuição uniforme é indicada quando a variância é pequena para todas as combinações de parcelas e o gráfico assemelha-se a uma linha reta e paralela ao eixo x. A interpretação dos gráficos ainda é subjetiva, mas geralmente a presença de picos nos blocos iniciais (tamanhos de bloco de 1, 2 e 3 ) ou em blocos que incluem em torno de 50\% do tamanho da transeção não deve ser considerada como indicações de agrupamentos de indivíduos (Campbell et al. 1998).

Considerando que é importante usar vários métodos para avaliar o padrão de distribuição espacial ou a escala de uma espécie, os dados foram analisados através de três métodos da variância: variância entre blocos de parcelas $(\mathrm{BQV})$; variância móvel entre blocos de parcelas (TTLQV) e variância entre parcelas pareadas (PQV). Todas as análises foram feitas usando os programas fornecidos em Ludwig \& Reynolds (1988). Estes autores sugerem que o número de blocos analisados nos métodos TTLQV e PQV não devem passar de 10 a $20 \%$ do total da extensão da transeção, entretanto, na maioria dos estudos que utilizam estes métodos, resultados são interpretados até a metade da distância total da transeção (Campbell et al. 1998).

Para as espécies arbóreas, duas análises foram feitas, a primeira utilizando os dados de todos os indivíduos encontrados dentro das 128 parcelas e a segunda utilizando somente os indivíduos com altura maior que $130 \mathrm{~cm}$. Esta segunda análise foi realizada para testar se os indivíduos maiores tiveram o mesmo padrão observado para o conjunto total de indivíduos. Para as palmeiras foi possível coletar dados somente nas primeiras 84 parcelas da transeção. Consequentemente, a análise BQV foi feita utilizando os dados das primeiras 64 parcelas, mas as análises do TTLQV e PQV foram realizadas utilizando os dados de todas as 84 parcelas. Também foi calculado o índice de dispersão para cada espécie, usando todos os indivíduos encontrados.

\section{Resultados e Discussão}

Das espécies lenhosas estudadas, $C$. brasiliense teve a maior densidade e $P$. pubescens a menor (tabela 1). Para todas as espécies, o número de indivíduos por parcela geralmente foi baixo $(\leq 2)$, porém $P$. pubescens teve densidade alta em algumas parcelas (até 12 indivíduos). Quando somente os indivíduos maiores foram analisados, $S$. paniculatum apresentou a densidade maior e poucos indivíduos de $P$. pubescens foram encontrados dentro das parcelas. Entre as palmeiras, S. flexuosa teve densidade maior, porém, em nenhuma das parcelas, foram encontrados mais de três indivíduos.

Estudos prévios sobre a distribuição espacial de espécies arbóreas e arbustivas no cerrado utilizaram os métodos clássicos para a determinação do padrão de distribuição espacial em parcelas, tais como: o índice de dispersão (Dusi 1989, Oliveira et al. 1989, Meirelles \& Luiz 1995); o índice de Morisita (Dusi 1989, Meirelles \& Luiz 1995) ou outros métodos como o índice de grau de agregação de MacGuinnes (Salomão \& Leite 1993). Destes estudos, a maioria calculou o padrão para uma espécie só. O único que avaliou o padrão para mais do que três foi o de Meirelles \& Luiz (1995), que apresentou dados para 18 espécies. Especificamente para as espécies $C$. brasiliense, $S$. paniculatum, S. comosa e $S$. flexuosa é possível obter dados de outros estudos para comparar com os resultados obtidos neste estudo (tabela 2). A comparação destes resultados mostra consistência quanto ao tipo de padrão encontrado para cada espécie. As diferenças provavelmente são relacionadas com o tamanho da área levantada e número total de indivíduos encontrados, no caso de Silva (1990) e o número reduzido de indivíduos de $S$. comosa encontrados no presente estudo.

A análise dos resultados usando a técnica $\mathrm{BQV}$ (figura 1) mostrou padrões diferentes entre as espécies arbóreas quando todos os indivíduos nas parcelas foram analisados, não havendo coincidência entre picos. Para $C$. brasiliense a distância entre grupos de indivíduos foi estimada em $320 \mathrm{~m}$, para $S$. paniculatum em $160 \mathrm{~m}$ e para $P$. pubescens não houve uma definição clara de picos. Um resultado

Tabela 1. Número total de parcelas levantadas $(8 \times 5 \mathrm{~m})$ e de indivíduos encontrados, número de indivíduos/parcela e variância para as cinco espécies estudadas no cerrado da Fazenda Água Limpa, Distrito Federal.

\begin{tabular}{|c|c|c|c|c|c|c|c|c|}
\hline & \multicolumn{2}{|c|}{ C. brasiliense } & \multicolumn{2}{|c|}{ P. pubescens } & \multicolumn{2}{|c|}{ S. paniculatum } & \multirow{2}{*}{$\frac{\text { S. comosa }}{\text { Todos }}$} & \multirow{2}{*}{$\frac{\text { S. flexuosa }}{\text { Todos }}$} \\
\hline & Todos & $>130$ & Todos & $>130$ & Todos & $>130$ & & \\
\hline Parcelas levantadas & 128 & 128 & 128 & 128 & 128 & 128 & 84 & 84 \\
\hline Número total de indivíduos & 138 & 50 & 34 & 9 & 85 & 62 & 8 & 36 \\
\hline Indivíduos/parcela & 1,08 & 0,39 & 0,27 & 0,07 & 0,66 & 0,48 & 0,095 & 0,428 \\
\hline Variância & 1,46 & 0,41 & 2,02 & 0,16 & 0,84 & 0,49 & 0,334 & 0,810 \\
\hline
\end{tabular}

Todos: Conjunto total de indivíduos encontrados; > 130: Indivíduos com altura superior a $130 \mathrm{~cm}$. 
Tabela 2. Resumo dos dados disponíveis sobre a distribuição espacial das cinco espécies do cerrado estudadas. ID = Índice de dispersão Mor = Índice de Morisita; C-E = Índice de Clark-Evans.

\begin{tabular}{lcll}
\hline Espécie & Método & Resultado & \multicolumn{1}{c}{ Referência } \\
\hline Caryocar brasiliense & ID & Agrupado* & Silberbauer-Gottsberger \& Eiten 1987 \\
Caryocar brasiliense & ID & Aleatório* & Silva 1990 \\
Caryocar brasiliense & Mor, ID & Agrupado & Miranda 1992 \\
Caryocar brasiliense & C-E & Agrupado & J. D. Hay, dados não publicados \\
Caryocar brasiliense & ID & Agrupado & Este estudo \\
Pterodon pubescens & ID & Agrupado & Este estudo \\
Sclerolobium paniculatum & ID & Aleatório* & Silva 1990 \\
Sclerolobium paniculatum & ID & Agrupado & Miranda 1992 \\
Sclerolobium paniculatum & Mor, ID & Agrupado & Meirelles \& Luiz 1995 \\
Sclerolobium paniculatum & C-E & Agrupado & J. D. Hay, dados não publicados \\
Sclerolobium paniculatum & ID & Agrupado & Este estudo \\
Syagrus comosa & ID, Mor & Agrupado & Dusi 1989 \\
Syagrus comosa & ID & Aleatório & Este estudo \\
Syagrus flexuosa & ID, Mor & Agrupado & Dusi 1989 \\
Syagrus flexuosa & ID & Agrupado & Este estudo \\
\hline
\end{tabular}

* O padrão de distribuição espacial foi calculado baseado nos dados apresentados no texto do artigo citado, usando uma parcela de tamanho $10 \times 10 \mathrm{~m}$.

semelhante foi encontrado para o conjunto de indivíduos com altura $>130 \mathrm{~cm}$, mas para estes indivíduos a espécie $C$. brasiliense também apresentou um pico para a distância de $40 \mathrm{~m}$. O padrão de agrupamento de $S$. flexuosa foi bem mais intenso, mostrando um forte padrão de agrupamento a intervalos de $80 \mathrm{~m}$, do que o padrão de $S$. comosa, que teve um pico baixo para a distância de $40 \mathrm{~m}$.

Usando o método TTLQV (figura 2), $C$. brasiliense e $S$. paniculatum mostraram um padrão de agrupamento em escala maior, com distância entre os grupos de 340 e $410 \mathrm{~m}$ respetivamente. $P$. pubescens apresentou um pico no início do gráfico, indicando uma distância na ordem de $40 \mathrm{~m}$ entre grupos. Para os indivíduos maiores que $130 \mathrm{~cm}$ de altura, $C$. brasiliense apresentou um padrão com picos pequenos, mas com indicação de grupo em torno de $350 \mathrm{~m}$. S. paniculatum mostrou o mesmo padrão visto para o conjunto de todos os indivíduos, com distância de $350 \mathrm{~m}$ estimada entre grupos. $P$. pubescens não mostrou picos, provavelmente devido ao baixo número de indivíduos disponível para esta análise. Entre as palmeiras, somente $S$. flexuosa mostrou um padrão com um pico, indicando uma distância de $80 \mathrm{~m}$ entre grupos.
Em muitos casos, os resultados do método PQV são difíceis de interpretar (Dantas 1994) e para os resultados obtidos com as espécies neste estudo isto parece verdade também. Em poucos casos, os gráficos deste método apresentaram picos bem nítidos. No método PQV (figura 3), $C$. brasiliense apresentou um padrão com picos baixos, mas em número maior que nos outros métodos, indicando grupos com distâncias de 180, 300 e 490 m entre seus centros. S. paniculatum apresentou picos baixos, mostrando um padrão essencialmente aleatório. $P$. pubescens mostrou dois picos, com uma distância de 110 e 440 m entre os centros. Na análise dos indivíduos com altura superior a $130 \mathrm{~cm}, C$. brasiliense mostrou dois picos, com centros distantes 40 e $150 \mathrm{~m}, S$. paniculatum mostrou um pico, indicando uma distância de $200 \mathrm{~m}$ entre grupos e $P$. pubescens não mostrou picos. Entre as palmeiras, somente $S$. flexuosa mostrou um pico neste método, indicando uma distância de 70 m entre os centros dos grupos.

Quando comparam-se os métodos, há uma certa correspondência quanto à localização dos picos. $C$. brasiliense mostrou picos com aproximadamente 300 a 350 m de distância entre os centros, tanto para 

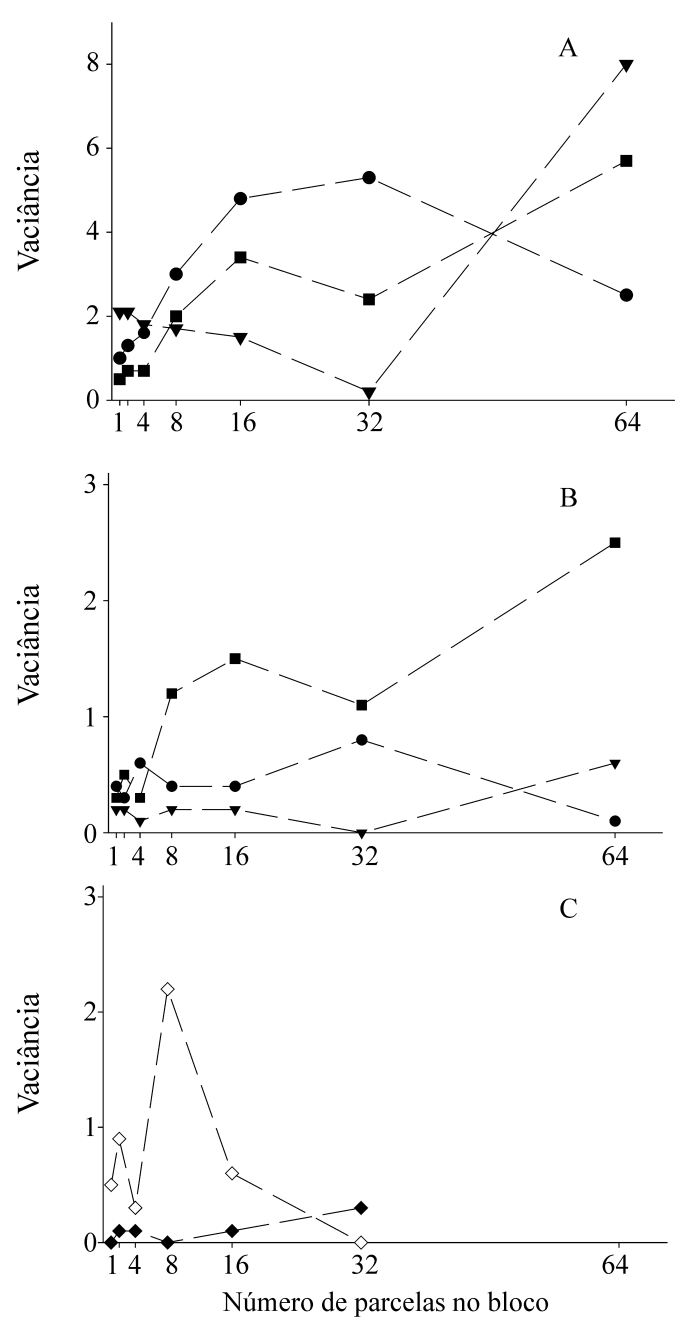

Figura 1. Análise do padrão de distribuição espacial de espécies nativas numa área de cerrado na Fazenda Água Limpa, Brasília, DF, usando o método de variância entre blocos de parcelas (BQV). A. Para todos os indivíduos das espécies arbóreas Caryocar brasiliense (--), Pterodon pubescens (- $\mathbf{\nabla}$-) e Sclerolobium paniculatum (-口-); B. Para indivíduos de Caryocar brasiliense (-0-),

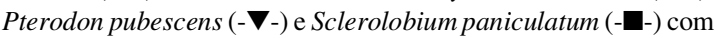
altura maior do que $130 \mathrm{~cm}$; C. Para as palmeiras Syagrus comosa $(-\downarrow-)$ e Syagrus flexuosa (-৩-).

todos os indivíduos levantados quanto para os indivíduos maiores que $130 \mathrm{~cm}$ de altura. Este resultado é semelhante ao de Dantas (1994) para esta espécie no cerrado de Minas Gerais. P. pubescens não mostrou tanta consistência entre métodos, mas teve uma tendência para indicação de grupos com distância relativamente curta entre centros, de 40 a
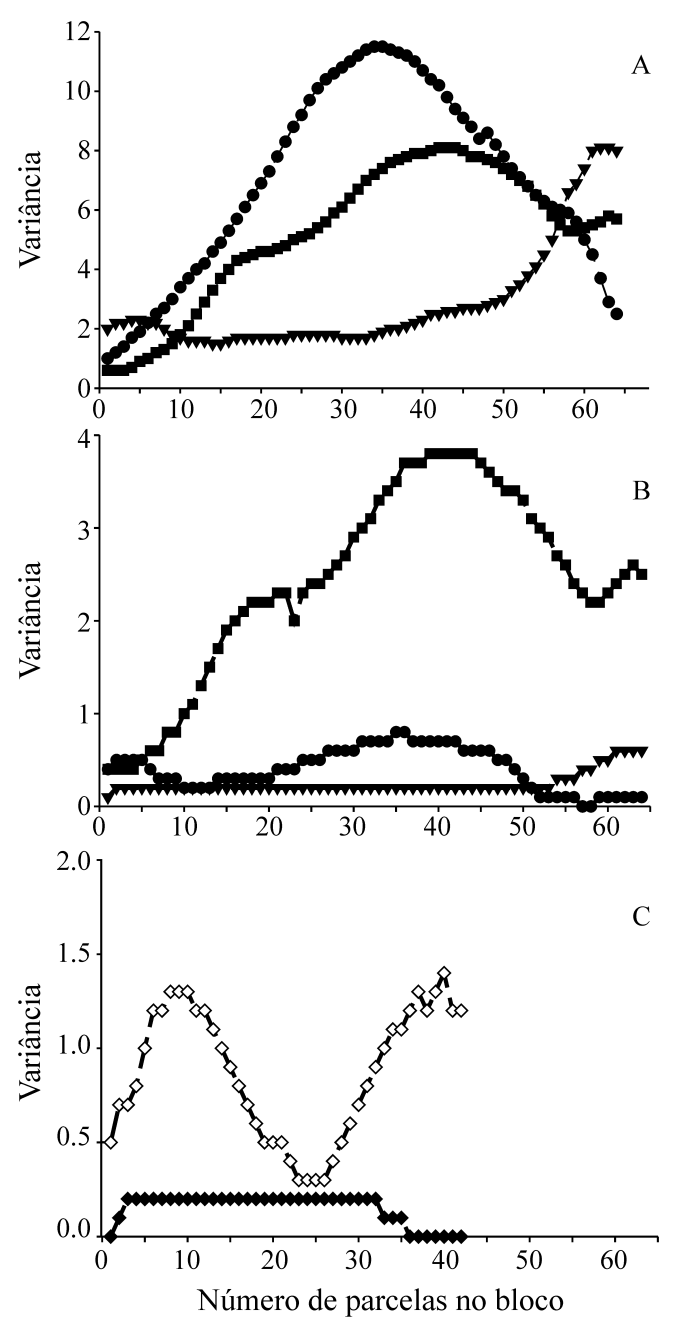

Figura 2. Análise do padrão de distribuição espacial de espécies nativas numa área de cerrado na Fazenda Água Limpa, Brasília, DF, usando o método de variância móvel entre blocos de parcelas (TTLQV) A. Para todos os indivíduos das espécies arbóreas

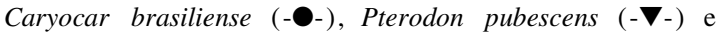
Sclerolobium paniculatum (-口-); B. Para indivíduos de Caryocar brasiliense (-0), Pterodon pubescens (-ם-) e Sclerolobium paniculatum (-) com altura maior do que $130 \mathrm{~cm}$; C. Para as palmeiras Syagrus comosa (- -) e Syagrus flexuosa $\left(-\diamond_{-}\right)$.

110 m. Para S. paniculatum, os métodos BQV e PQV apresentaram resultados semelhantes, com distâncias de 160 a $200 \mathrm{~m}$ entre os centros dos grupos, mas a análise com o método TTLQV indicou o dobro deste valor, com uma distância em torno de $400 \mathrm{~m}$. No caso da palmeira $S$. flexuosa, os métodos foram consistentes, indicando uma distância de aproxi- 

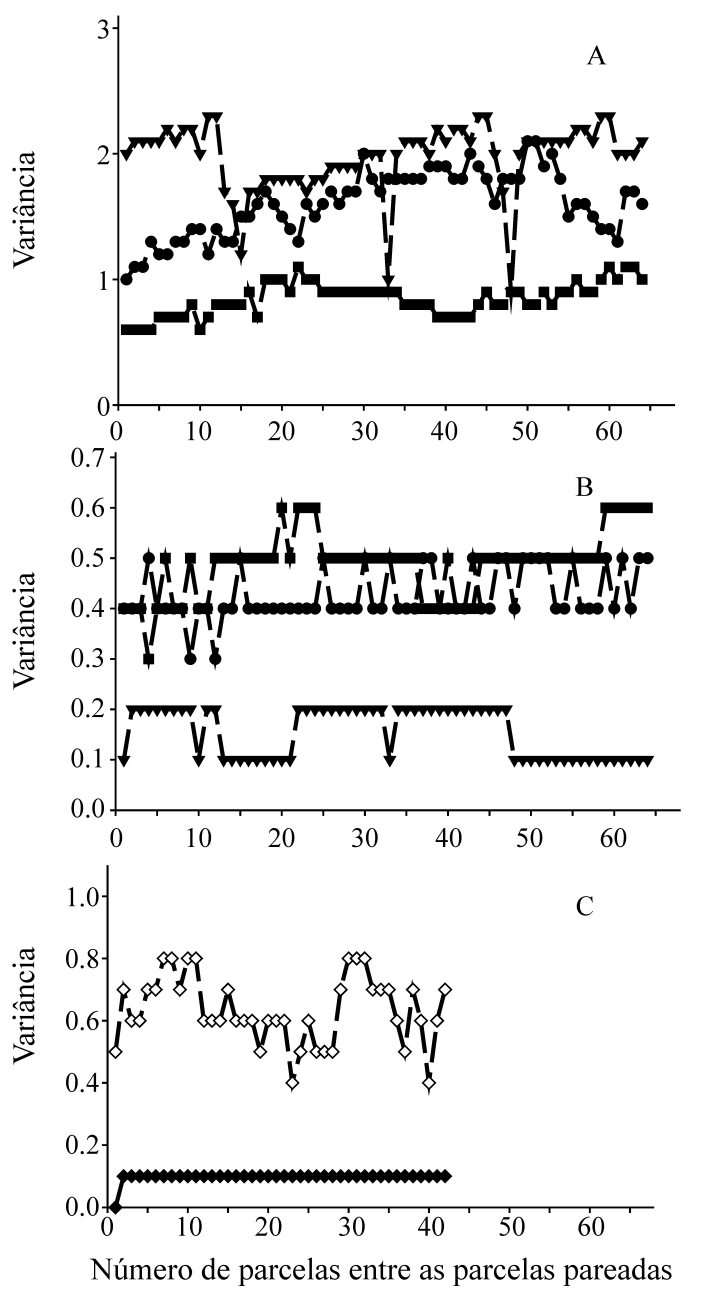

Figura 3. Análise do padrão de distribuição espacial de espécies nativas numa área de cerrado na Fazenda Água Limpa, Brasília, $\mathrm{DF}$, usando o método de variância entre parcelas pareadas (PQV). A. Para todos os indivíduos das espécies arbóreas Caryocar

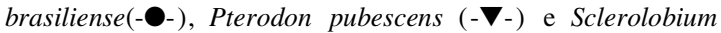
paniculatum (--); B. Para indivíduos de Caryocar brasiliense

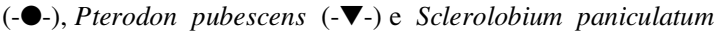
(-口-) com altura maior que $130 \mathrm{~cm}$; C. Para as palmeiras Syagrus comosa (- -) e Syagrus flexuosa (-৩-).

madamente 70 a $80 \mathrm{~m}$ entre os centros dos grupos. A similaridade ou diferença na localização dos picos observados para cada espécie não deve ser interpretada como uma associação positiva ou negativa entre estas espécies, pois, para isso é necessário outro tipo de análise (Coomes et al. 1999).

Alguns outros estudos de espécies do cerrado procuraram diferenças no padrão ou grau de agru- pamento de indivíduos jovens e adultos da mesma espécie (Oliveira et al. 1989, Santos 1991), mas os resultados destes estudos não foram consistentes. No presente estudo, observa-se, para $C$. brasiliense, a presença de picos, embora de pouca intensidade, indicando distância menor na análise de indivíduos com altura $130 \mathrm{~cm}$. Isto indica um certo grau de agrupamento destes indivíduos maiores. Espera-se, para espécies com diásporos pesados, como $C$. brasiliense, ou espécies com crescimento vegetativo, como $P$. pubescens, um padrão de distribuição agrupada mais intensa em escala menor. Uma possível explicação para este resultado seria que os indivíduos maiores estariam localizados em manchas ambientais propícias para o desenvolvimento da espécie, enquanto os indivíduos menores estariam mais espalhados na comunidade e ainda não sofreram mortalidade. Em uma savana na África, GriegSmith (1991) aplicou um método de variância em conjuntos de espécies selecionadas por circunferência. Os resultados deste estudo, em geral, indicaram um padrão semelhante em todas as classes. De qualquer forma, um estudo sobre a distribuição espacial de uma espécie deve incluir indivíduos de todos os tamanhos. É necessário perguntar, no entanto, qual é a distância entre grupos de indivíduos dentro da comunidade, ou seja, qual é o tamanho do grão para cada espécie. Os métodos de análise ora utilizados podem ajudar a responder esta pergunta.

Em suma, os métodos utilizados mostraram-se eficientes e o tempo gasto na coleta de dados foi compatível com outros métodos usados para determinação do padrão de distribuição espacial. As espécies estudadas mostraram padrões de agrupamento em escalas diferentes e os métodos empregados apresentaram resultados semelhantes entre si para três das cinco espécies. Para $C$. brasiliense, houve semelhança entre os resultados obtidos neste estudo e em outro em que se empregou a mesma metodologia relacionada ao tamanho de mancha desta espécie.

Agradecimentos - Os autores agradecem ao Dr. Fernando Dantas, pelos dados referentes a Caryocar brasiliense e pela discussão da metodologia. 


\section{Referências bibliográficas}

BROWER, J.E., ZAR, J.H. \& von Ende, C.A. 1998. Field and laboratory methods for general ecology. 4 ed. Wm. C. Brown Publishers, Dubuque.

CAMPBELL, J.E., FRANKLIN, S.B., GIBSON, D.J. \& NEWMAN, J.A. 1998. Permutation of two-term local quadrat variance analysis: General concepts for interpretation of peaks. Journal of Vegetation Science 9:41-44.

CARTER, A.J. \& O'CONNOR, T.G. 1991. A 2-phase mosaic in a savanna grassland. Journal of Vegetation Science 2:231-236.

COOMES, D.A., REES, M. \& TURNBULL, L. 1999. Identifying aggregation and association in fully mapped spatial data. Ecology 80:554-565.

DANTAS, F. 1994. The ecology, ethnobotany and management of Caryocar brasiliense Camb. around Montes Claros, MG, Brazil. Ph.D. thesis, University of Oxford, Oxford.

DUSI, R.L.M. 1989. Padrão espacial, estrutura de populações e associação de Syagrus comosa Mart. e S. flexuosa Mart. (Palmae) em um cerrado do Jardim Botânico de Brasília, DF. Dissertação de mestrado, Universidade de Brasilia, Brasília.

GOODALL, D.W. 1974. A new method for the analysis of spatial pattern by random pairing of quadrats. Vegetatio 29:135-146.

GRIEG-SMITH, P. 1952. The use of random and contiguous quadrats in the study of the structure of plant communities. Annals of Botany 16:293-316.

GRIEG-SMITH, P. 1991. Pattern in a derived savanna in Nigeria. Journal of Tropical Ecology 7:491-502.

HUBBELL, S.P. \& FOSTER, R.B. 1986. Commoness and rarity in a neotropical forest: implications for tropical tree conservation. In Conservation biology: science of scarcity and diversity (M.E. Soule, ed.). Sinauer Associates Inc., Sunderland, p.205-231.

LEVIN, S.A. 1992. The problem of pattern and scale in ecology. Ecology 73:1943-1967.

LUDWIG, J.A. \& GOODALL, D.W. 1978. A comparison of paired- with blocked-quadrat methods for the analysis of spatial patterns. Vegetatio 38:49-59.

LUDWIG, J.A. \& REYNOLDS, J.F. 1988. Statistical ecology: a primer on methods and computing. John Wiley \& Sons, New York.
MEIRELLES, M.L. \& LUIZ, A.J.B. 1995. Padrões espaciais de árvores de um cerrado em Brasília, DF. Revista Brasileira de Botânica 18:185-189.

MIRANDA, I.S. 1992. Estrutura e fenologia de uma comunidade arbórea da savana amazônica de Alter-do-Chão, Pará. Dissertação de mestrado, Fundação Universidade da Amazônia, Manaus.

OLIVEIRA, P.E.A.M., RIBEIRO, J.F. \& GONZALES, M.I. 1989. Estrutura e distribuição espacial de uma população de Kielmeyera coriacea Mart. de cerrados em Brasília. Revista Brasileira de Botânica 12:39-47.

PIELOU, E.C. 1969. An introduction to mathematical ecology. Wiley-Interscience, New York.

RATTER, J.A., BRIDGEWATER, S., ATKINSON, R. \& RIBEIRO, J.F. 1996. Analysis of the floristic composition of the Brazilian cerrado vegetation: II. Comparison of the woody vegetation of 96 areas. Edinburgh Journal of Botany 53:153-180

SALOMÃO, A.N. \& LEITE, A.M.C. 1993. Comportamento de regenerantes de Astronium urundeuva (Fr. All.) Engler em área sob ação antrópica. Acta Botânica Brasilica 6:85-96.

SAN JOSE, J.J., FARINAS, M.R. \& ROSALES, J. 1991. Spatial patterns of trees and structuring factors in a Trachypogon savanna of the Orinoco Llanos. Biotropica 23:114-123.

SANTOS, F.A.M. 1991. Padrão espacial de jovens em relação a adultos de espécies arbóreas de cerrado que ocorrem no Estado de São Paulo. Tese de doutorado, Universidade Estadual de Campinas, Campinas.

SCHAEFER, J.A. 1993. Spatial patterns of tiaga plant-communities following fire. Canadian Journal of Botany 71:1568-1573.

SILBERBAUER-GOTTSBERGER, I. \& EITEN, G. 1987. A hectare of cerrado. I. General aspects of the trees and thick-stemmed shrubs. Phyton 27:55-91.

SILVA, F.C. 1990. Compartilhamento de nutrientes em diferentes componentes da biomassa aérea em espécies de um cerrado. Dissertação de mestrado, Universidade de Brasilia, Brasília, DF.

STOHLGREN, T.J. 1993. Spatial patterns of giant sequoia (Sequoiadendron giganteum) in 2 sequoia groves in Sequoia National Park, California. Canadian Journal of Forest Research 23:120-132. 